J3eA, Journal sur l'enseignement des sciences et technologies de l'information et des systèmes, Volume 4, Hors-Série 3, 5 (2005)

DOI : http://dx.doi.org/10.1051/bib-j3ea:2005805

(C) EDP Sciences, 2006

RAVIR : des systèmes de vision active pour le relevé anatomique 3D appliqué à la santé

\author{
Y. Lucas ${ }^{1}$, S. Treuillet ${ }^{1}$, B. Albouy ${ }^{1}$ et J.C. Pichaud ${ }^{2}$ \\ ${ }^{1}$ Laboratoire Vision et Robotique, Université d'Orléans, France \\ ${ }^{2}$ Centre de Médecine Physique et de Réadaptation, F-47200 Virazeil, France
}




\title{
RAVIR : Des Systèmes de Vision Active pour le Relevé Anatomique 3D Appliqué à la Santé
}

\author{
Y. Lucas ${ }^{1}$, S.Treuillet ${ }^{1}$, B.Albouy ${ }^{1}$, Dr J.C.Pichaud ${ }^{2}$ \\ ${ }^{1}$ Laboratoire Vision et Robotique, Université d'Orléans. \\ ${ }^{2}$ Centre de Médecine Physique et de Réadaptation 47200 Virazeil \\ yves.lucas@bourges.univ-orleans.fr, sylvie.treuillet@univ-orleans.fr, benjamin.albouy@ensi-bourges.fr, \\ jcpichaud@wanadoo.fr
}

Résumé : Au travers du programme RAVIR dédié aux relevés anatomique du corps humain, on aborde ici la spécificité des systèmes de vision active orientés vers la santé. Longtemps limités aux applications industrielles, ces systèmes révolutionnent des pratiques médicales manuelles par une numérisation sans contact et le traitement informatique des données 3D. Trois applications biomédicales sont présentées : le dépistage des scolioses, la conception de semelles orthopédiques et le suivi thérapeutique des escarres. Les choix techniques propres à chaque application sont commentés en suivant les étapes clé de la conception d'un système de vision active.

Mots clés : vision active, reconstruction 3D, biomédical.

\section{Relevés anatomiques}

Le corps humain comme sujet de mesure est l'objet de la biométrie où la vision artificielle résoud déjà des problèmes d'identification liés à la sécurité: reconnaissance automatique de visages, d'empreintes digitales, de la pupille oculaire [1-2]...mais aussi pour l'industrie de l'habillement [3] ou de la création cinématographique [4 -5]. Dans le domaine médical ,la pratique courante de relevé anatomique reste le moulage. Tout traitement informatique de ces empreintes figées est par nature impossible et la présence sur le terrain d'un spécialiste reste indispensable pour établir de visu un diagnostic. En marge des techniques d'exploration interne sophistiquées (scanner X, IRM, ultra-sons...), le relevé 3D de surface du corps humain est encore peu présent dans le monde de la santé. Pourtant, de nombreux systèmes existent depuis longtemps pour le prototypage de pièces manufacturées, avec des passerelles vers les logiciels de CFAO.

L'objectif de RAVIR (Relevés Anatomiques par Vision laseR) est le développement de systèmes d'imagerie 3D, capables de numériser instantanément une région anatomique et d'offrir de nouveaux outils pour le diagnostic et le suivi des patients. Trois applications cibles sont développées actuellement (Fig.1). Le système SYDESCO (SYstème de DEpistage des SCOlioses) a été mis en œuvre pour le dépistage de masse en milieu scolaire des scolioses par vision active [6]. Le système OPAL (Orthèse Plantaire Assistée par Laser) vise la conception de semelles orthopédiques [7]. Enfin, le projet ESCALE (EScarre Analyse Lisibilité Evaluation) a pour objectif le suivi thérapeutique d'escarres par vision couleur non calibrée [8].

La conception d'un système de vision active adapté au relevé d'une région anatomique comprend plusieurs étapes: choix de la technique de projection de lumière structurée et de l'ergonomie du système, méthode de calibrage, détection du faisceau dans l'image, et enfin analyse des données 3D et application clinique. Chaque système conduira à des choix bien spécifiques pour chacune de ces étapes 
que nous allons décrire ci-dessous. Mais nous donnons pour commencer un état des lieux sur les techniques de mesure anatomiques très rudimentaires avant l'introduction du relevé par vision active.

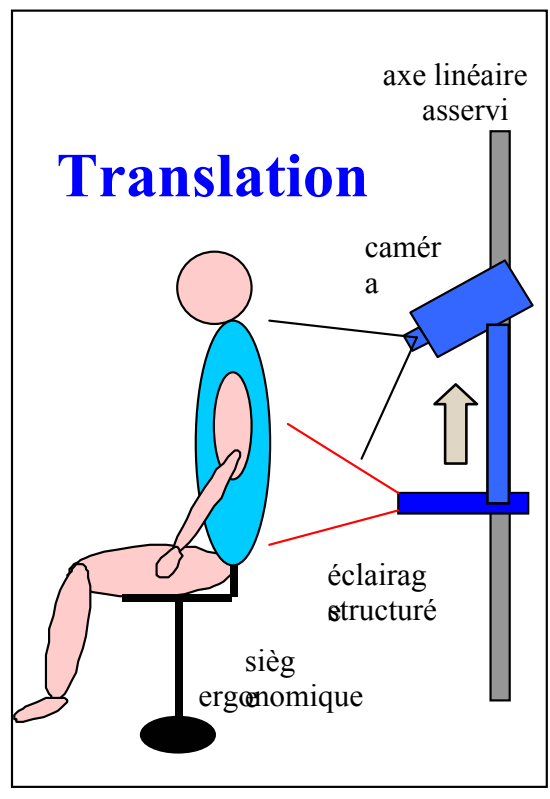

(a)

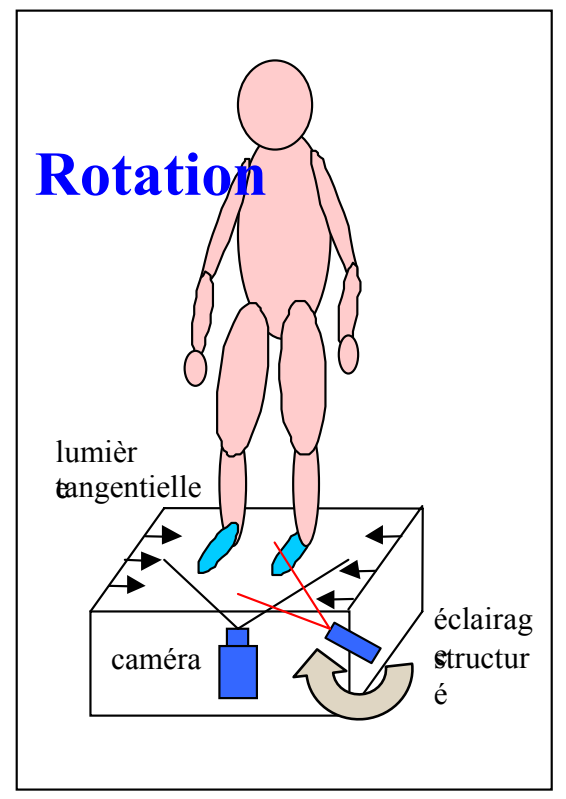

(b)

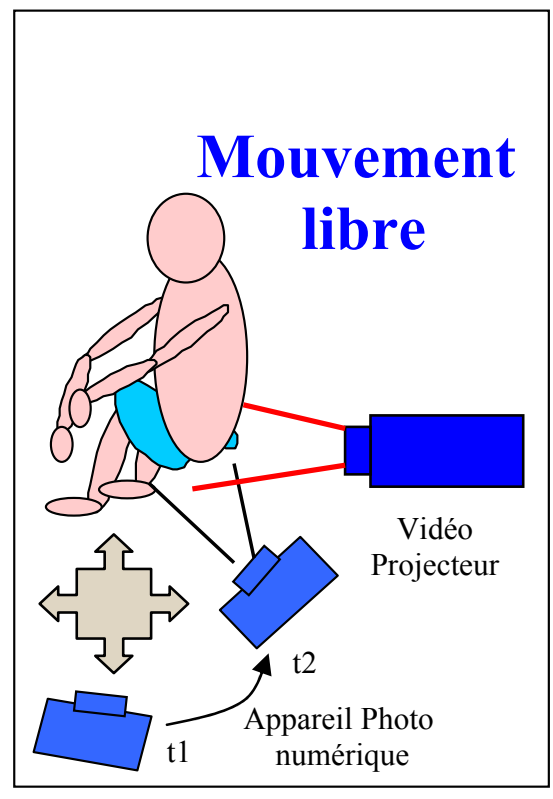

(c)

Figure 1 - Systèmes de vision pour le relevé anatomique (a) cabine de dépistage de scolioses (b) podoscope instrumenté (c) relevé d'escarres par appareil photo numérique

\section{Pratiques cliniques}

Force est de constater que les techniques de numérisation du corps humain sont restées longtemps artisanales dans les centres hospitaliers, alors que l'imagerie fonctionnelle s'était déjà dotée d'équipements high-tech comme les scanner de tomographie $\mathrm{X}$, la résonance magnétique nucléaire ou encore l'échographie :

- pour détecter et suivre une pathologie du rachis, un examen médical effectué par un médecin et complété systématiquement de radiographies (Fig.2a) demeure une pratique courante. Or, pendant la croissance chez l'adolescent, des scolioses très évolutives peuvent se développer. Une raison valable pour développer une cabine ambulante de dépistage de masse des scolioses en milieu scolaire. Toute dissymétrie du rachis (Fig.2b) est détectée automatiquement et l'enfant peut être orienté vers un spécialiste. De même, pendant la rééducation où le port d'un corset est préconisé (Fig.2c), celui-ci était auparavant réalisé de façon artisanale d'après un moulage direct sur le dos du patient. Là encore, le relevé anatomique 3D fournit la géométrie d'un corset et permet un suivi thérapeutique de la région dorsale du patient par des relevés successifs. 


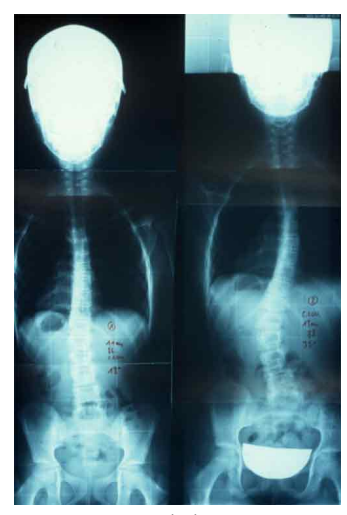

(a)

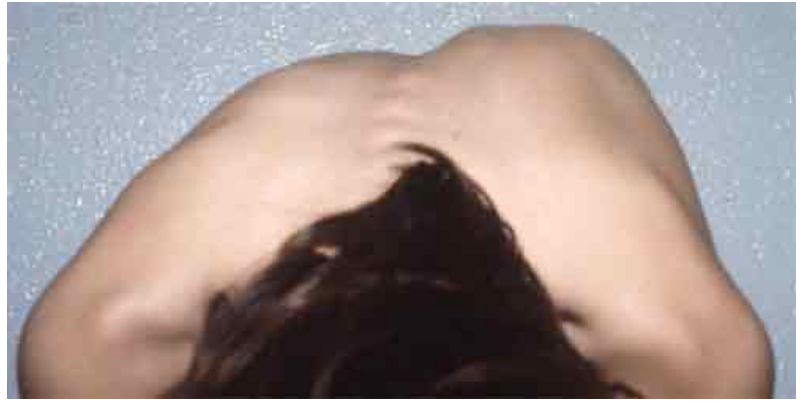

(b)

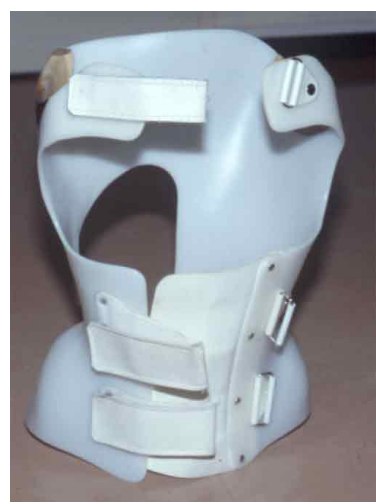

(c)

Figure 2 - radiographie X d'une scoliose (a) dissymétrie scoliotique ou gibbosité (b) corset (c)

- lors d'une consultation de podologie, le spécialiste fait monter le patient sur un podoscope et visualise l'empreinte des appuis plantaires grâce à un miroir placé sous la vitre et un éclairage rasant fluorescent. Mais cette empreinte 2D ne fait pas apparaître les zones sans contact avec la vitre, notamment la voûte plantaire, et doit être complété par un moulage pour produire une semelle orthopédique. Les techniques traditionnelles de mesure, empreinte sur papier carboné (Fig.3a), ou mieux moulage 3D se font dans le plâtre (Fig.3b), dans une mousse phénolique (Fig.3c) ou par thermoformage (Fig. 3d) (Conform'able-Sidas). Elles ne fournissent pas de modèle numérique du pied et interdisent donc tout traitement informatisé. Récemment, quelques systèmes onéreux sont apparus, essentiellement tournés vers les conception de chaussures de mode sur mesure (Yeti Foot scanner- Vorum Research Corp. Pedus 3-D - Techmath, Shoemaster-CSM3D ...) [9]. Notre objectif a été d'instrumenter un simple podoscope de cabinet médical avec un système de vision pour réaliser un scanner 3D plantaire bas coût, destiné à la confection de semelles monobloc en biomatériaux à but orthopédiques (personnes âgées) et ergonomiques (sportifs de haut niveau).

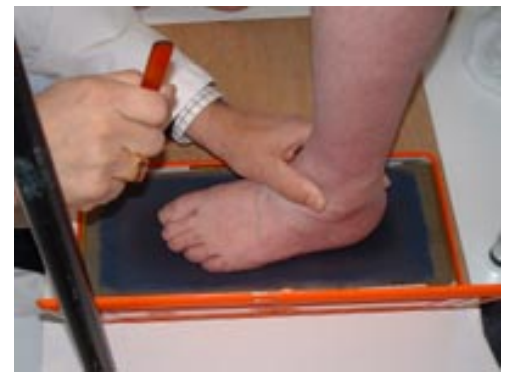

(a)

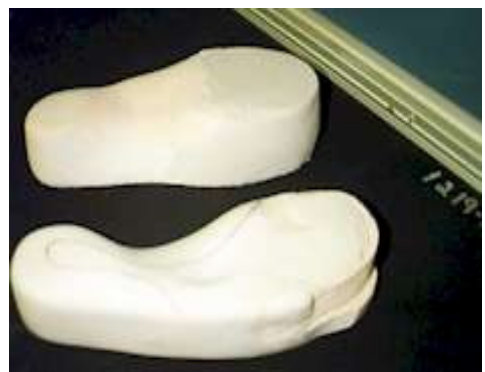

(b)

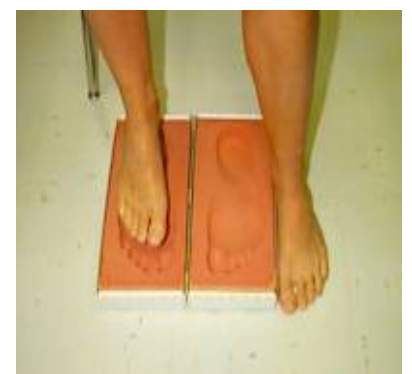

(c)

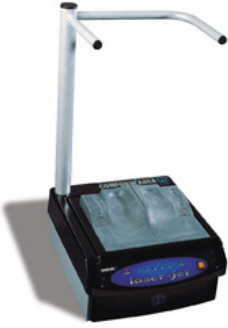

(d)

Figure 3 - relevé sur papier carboné (a) moulage en plâtre (b) empreinte sur mousse phénolique (c) thermoformage $(\mathrm{d})$

- pour évaluer le volume d'escarres, une pathologie très courante et très coûteuse pour le système de santé, les outils utilisés jusqu'à présent par les équipes cliniques sont très empiriques. Le personnel soignant se contente d'appréciations visuelles, d'esquisses, de recopie sur des calques, plus rarement de moulage à la pâte dentaire ou d'injection salines sous film plastique (Fig.4a). Outre le risque d'infection et la douleur occasionnée par l'examen, ces pratiques rendent aléatoires la coordination des soins lors des changements d'équipes médicales. Des prototypes de mesure 3D sont apparus récemment mais demeurent encombrants (Fig.4b), complexes à utiliser (Fig.4c) ou très coûteux (Fig.4d), ce qui interdit 
une diffusion massive dans les équipes de soin [10-13]. La prise de vue d'escarres sur des patients alités et handicapés nécessite un outil de prise de prise de vue très compact, ce qui nous a orienté vers une solution basée sur un simple appareil-photo numérique.

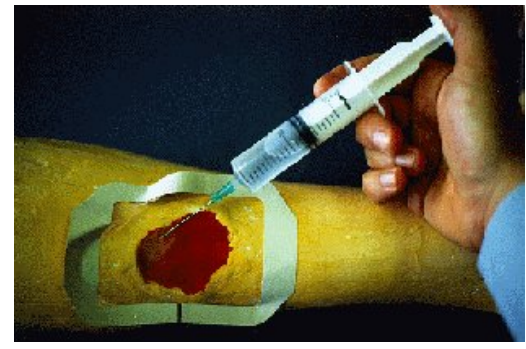

(a)

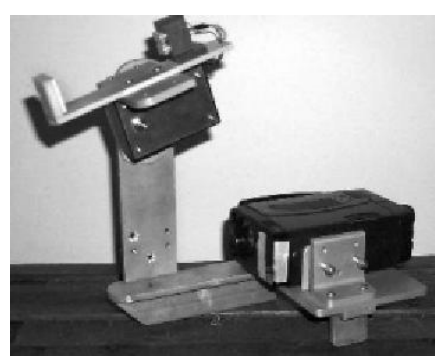

(b)

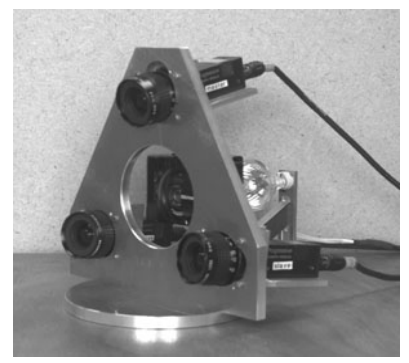

(c)

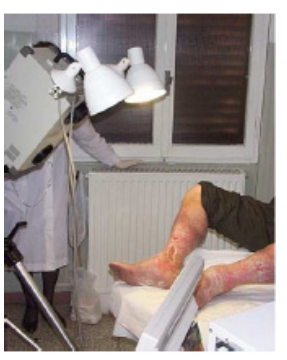

(d)

Figure 4 - injection saline sous film (a) vision active (b) stéréovision (c) système Minolta Vivid (d)

\section{Mode de projection et ergonomie}

En vision active, on exploite la triangulation entre un faisceau de lumière structurée et un imageur matriciel. Un éclairage structuré se compose de franges, de bandes ou de points associés à un codage spatial, temporel ou direct, qui permet d'identifier de façon unique chaque motif projeté [14].

Le motif projeté peut servir à la triangulation (Fig. 5a) ou juste ajouter de la texture sur l'objet (Fig. 5b). Plusieurs stratégies sont possibles pour gérer la projection du faisceau :

- L'objet peut-être déplacé devant le faisceau, soit par l'intermédiaire d'un plateau rotatif, soit par translation sur un convoyeur.

- Le faisceau, ponctuel ou plan, peut être déplacé par rotation ou translation pour scanner la pièce. On distingue alors les têtes de mesure montées en effecteur de machine à commande numérique (Fig. 6a), montées sur un pantin articulé muni de codeurs (Fig. 6b) ou celles dotées de capteurs magnétiques (Fig. 6c) pour une manipulation plus libre.

- La projection d'une image codée, composée de bandes ou de franges colorées dispense d'un balayage.

- La projection d'une image aléatoire dite de speckle peut aider la reconstruction 3D obtenue par stéréovision[15].

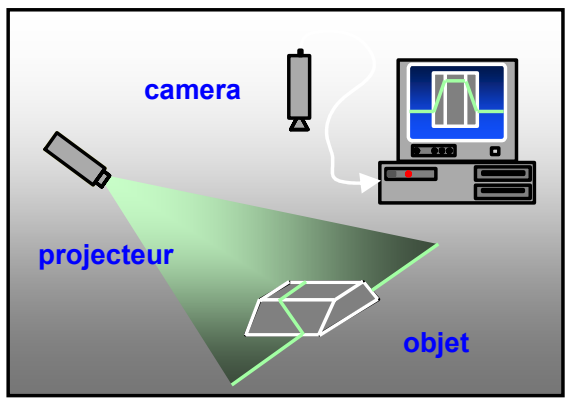

(a)

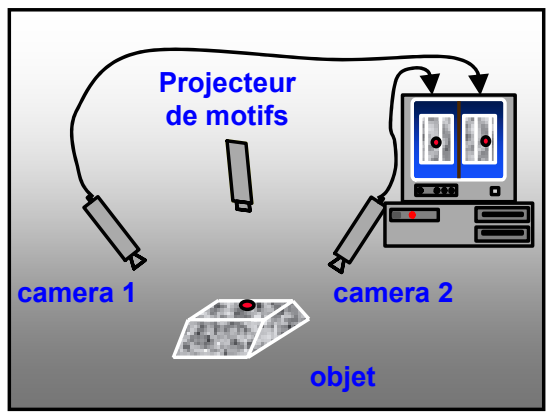

(b)

Figure 5 - triangulation avec un faisceau plan calibré (a) stéréovision renforcée par projection de lumière (b) 


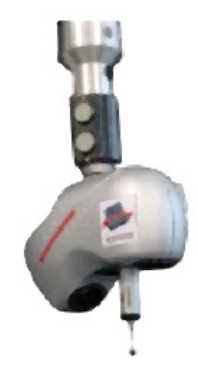

(a)

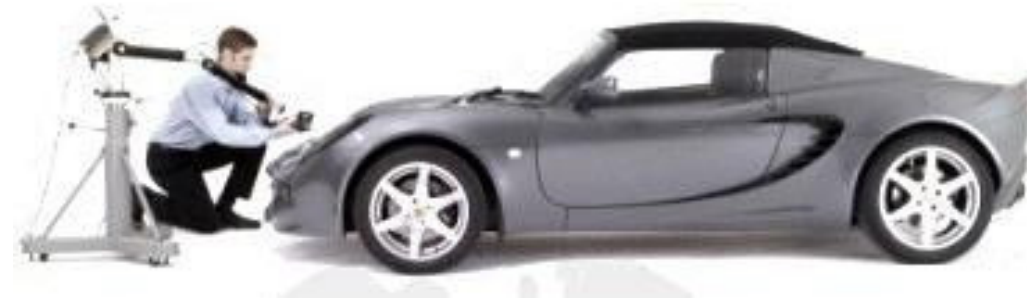

(b)

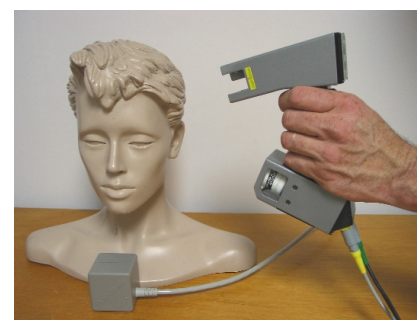

(c)

Figure 6 - tête Kréon sur machine CN (a) tête sur pantin (b) avec capteur magnétique 6DDL (c)

L'utilisation d'un système de vision active pour le relevé anatomique introduit des contraintes en termes d'ergonomie, de vitesse, d'espace de mesure et de sécurité, qui ont nous orienté vers des solutions spécifiques :

- pour un relevé dorsal, un tube HeNe générateur de ligne est intégré à une tête de mesure contenant aussi la caméra. Le déplacement vertical asservi en translation de toute la tête convient bien à la zone de mesure étendue et plane (scan en $2 \mathrm{sec}$ ).

- Le patient est assis sur un tabouret haut pour éviter toute attitude scoliotique simplement due à une inégalité de longueur des membres inférieurs. Le siège peut pivoter pour recaler le patient perpendiculairement au faisceau.

- dans le cas d'un relevé plantaire, la compacité du podoscope nous a conduit à dissocier la caméra du plan laser : la caméra observe la voûte plantaire par l'intermédiaire de miroirs placés au fond du podoscope (doublement de la distance de mise au point), et le faisceau plan d'une diode laser miniature est piloté en rotation par un moteur pas à pas haute résolution (scan en $1 \mathrm{sec}$ ).

- enfin, pour le relevé d'escarres, deux prises de vue libres sous des angles différents sont nécessaires pour reconstruire la plaie en 3D. La projection optionnelle d'une image aléatoire de speckle est utilisée pour rajouter de la texture sur des parties trop uniformes. La détection de points singuliers est alors plus robuste et plus dense sur la plaie.

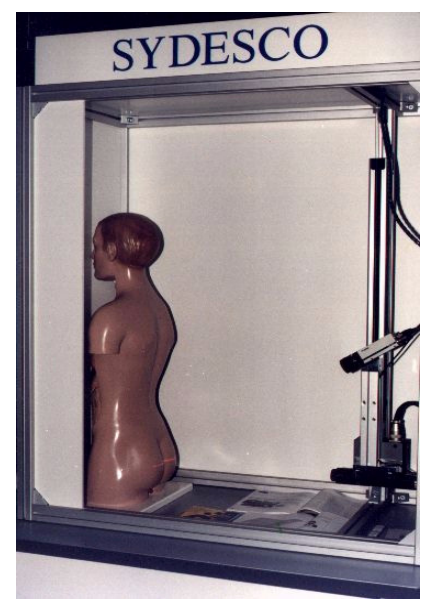

(a)

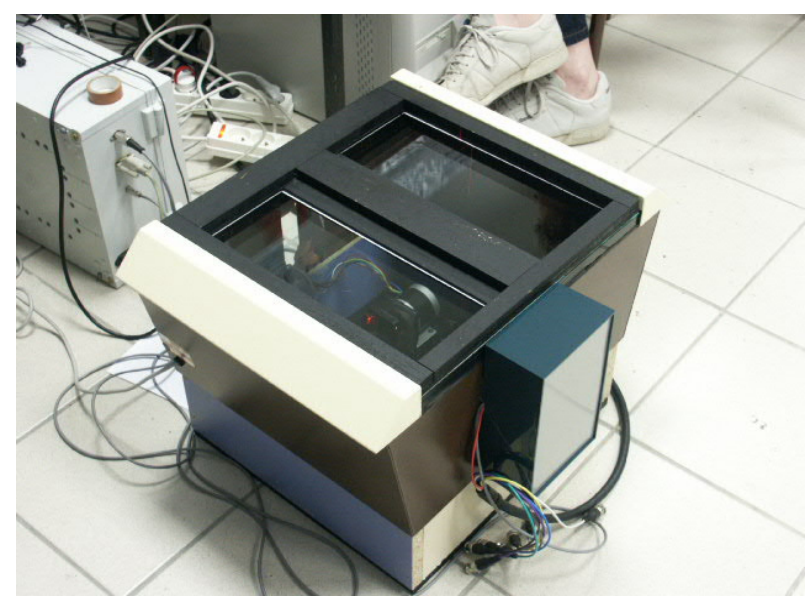

(b)

Figure 7 - cabine ambulante de dépistage des scolioses (a) podoscope instrumenté (b) 


\section{Calibrage}

Il conditionne la précision des mesures obtenues après reconstruction 3D. Lorsque la chaîne d'acquisition est simple à modéliser, on peut déterminer ses paramètres extrinsèques (position et orientation relative du plan laser et de la caméra) à partir de prises de vues d'un objet calibré [16-17].

- c'est le cas du relevé dorsal où caméra et plan laser sont rigidement liés dans la tête de mesure et se déplacent en translation (Fig. 8). La distance H séparant l'axe optique caméra du plan laser ainsi que l'inclinaison $\theta$ de ce plan laser avec l'axe optique sont les paramètres à calibrer. En exploitant par exemple l'image d'un profil laser oblique de longueur connue et d'extrémités $\mathrm{P}_{1}\left(\mathrm{x}_{\mathrm{C} 1}, \mathrm{z}_{\mathrm{c} 1}\right)$ et $\mathrm{P}_{2}\left(\mathrm{x}_{\mathrm{C} 2}\right.$, $\mathrm{Z}_{\mathrm{c} 2}$ ) dans l'image, on dispose d'une solution analytique simple. On obtient l'angle $\theta$ et la hauteur $\mathrm{H}$ par:

$$
\begin{aligned}
& \operatorname{tg} \theta=\left(x_{c 2}-x_{c 1}\right) /\left(z_{c 1}-z_{c 2}\right), 0<\theta<\pi / 2 \\
& H=x_{c 1} \cos \theta+z_{c 1} \sin \theta
\end{aligned}
$$

On en déduit les coordonnées $3 \mathrm{D}$ des points $\mathrm{P}\left(\mathrm{x}_{\mathrm{c}}, \mathrm{y}_{\mathrm{c}}, \mathrm{z}_{\mathrm{c}}\right)$ triangulés à partir de leur projection $\mathrm{P}^{\prime}(\mathrm{x}, \mathrm{y}$ ') dans l'image :

$$
x_{c}=\frac{H y^{\prime}}{f \sin \theta+y^{\prime} \cos \theta} \quad z_{c}=-\frac{x_{c} \cos \theta+H}{\sin \theta} \quad y_{c}=\frac{x^{\prime}}{f} z_{c}
$$

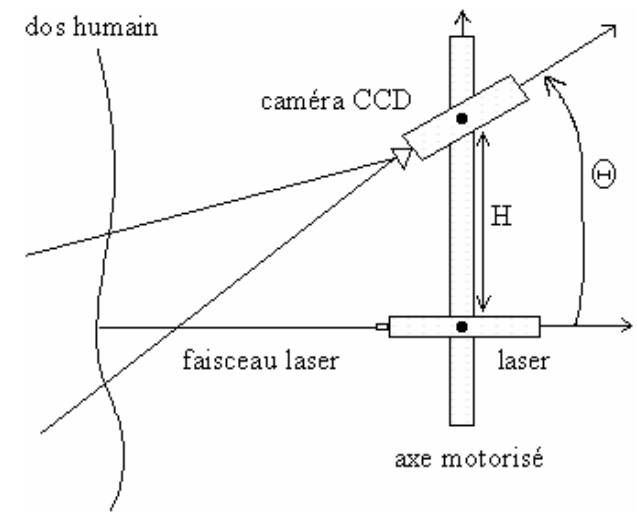

Figure 8 - tête de mesure pour le relevé 3D du rachis.

Des images de mire plane (damier) permettent de déterminer aussi les paramètres intrinsèques de la caméra (focale, taille des éléments CCD et distorsion)

- dans le cas du relevé plantaire, les phénomènes physiques sont plus complexes à modéliser (objectif grand-angle à forte distorsion, orientation caméra/laser variable, double réfraction avec la vitre du podoscope...). La zone de mesure étant très limitée spatialement ( l'arche plantaire ne se soulève que de quelques mm lorsque le pied est en charge), il est judicieux de procéder à un calibrage direct [18-19]. On s'affranchit de toute modélisation physique en recueillant un grand nombre de points 3D sur tout l'espace de mesure, intégrant toutes les imperfections telles que la distorsion optique. La reconstruction de forme s'appuie sur une interpolation de ce maillage de référence. Un étalonnage direct a été effectué sur douze plans en Z (environ 500000 points calibrés). Pratiquement, une mire plane est placée à différentes hauteurs au dessus du podoscope, sur des cales étalons et on recueille l'ensemble des profils 
obtenus pour un scan complet (Fig. 9a). Une interpolation est ensuite entre les plans de calibrage. L'observation des données de calibrage a montré que le décalage vertical d'un profil dans le plan image était proportionnel à la hauteur. Cette propriété permet de réduire le nombre de plans de calibrage, et d'estimer toute hauteur $\mathrm{Z}$ en $\mathrm{mm}$ d'un point du profil considéré par simple interpolation linéaire, avec la relation :

$$
\mathrm{Z}=\left(\mathrm{v}-\mathrm{h}_{\mathrm{n}+1}\right)\left(\mathrm{Z}_{\mathrm{n}+1}-\mathrm{Z}_{\mathrm{n}}\right) /\left(\mathrm{h}_{\mathrm{n}+1}-\mathrm{h}_{\mathrm{n}}\right)-\mathrm{Z}_{\mathrm{n}+1}
$$

où $\mathrm{v}\left(\mathrm{h}_{\mathrm{n}}<\mathrm{v}<\mathrm{h}_{\mathrm{n}+1}\right)$ désigne la position verticale en pixels du point observé dans l'image et $\mathrm{h}_{\mathrm{i}}$ la position verticale de référence dans l'image du profil considéré pour une hauteur calibrée $\mathrm{Z}_{\mathrm{i}}$.

Notons que la précision de mesure dépend de l'ergonomie du système et de l'échantillonnage (Fig. 9b). Dans le cas du podoscope, les profils ne sont pas espacés régulièrement lorsque le faisceau pivote d'un pas moteur et donc la résolution n'est pas homogène sur la zone de mesure. Ce problème est compensé par le fait que la sensibilité de mesure est meilleure en haut d'image pour des questions de triangulation. En effet, le décalage du profil laser dans l'image pour une variation locale $\Delta z$ du relief peut être estimé par la relation suivante où $\mathrm{f}$ désigne la focale caméra, $\mathrm{D}>>\Delta \mathrm{z}$, la distance caméra/élément de surface triangulé, $\phi$ l'angle entre le faisceau laser et l'axe optique :

$$
\mathrm{e}=(\mathrm{f} \Delta \mathrm{z} \sin \phi) /(\mathrm{D}+\Delta \mathrm{z} \cos \phi) \cong \mathrm{f} \Delta \mathrm{z} \sin \phi / \mathrm{D}
$$

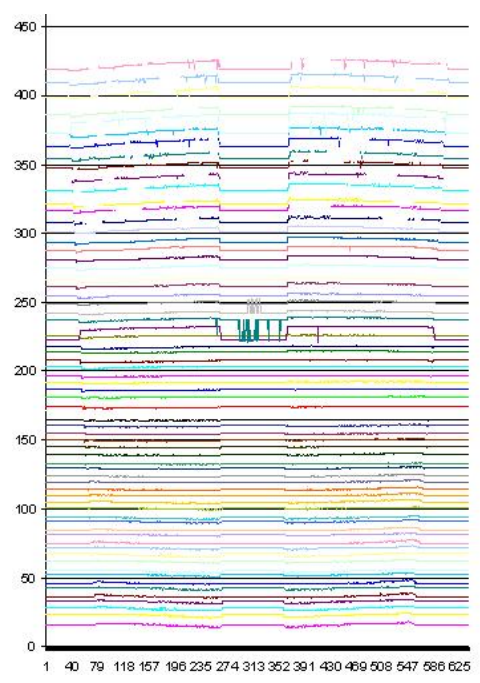

(a)

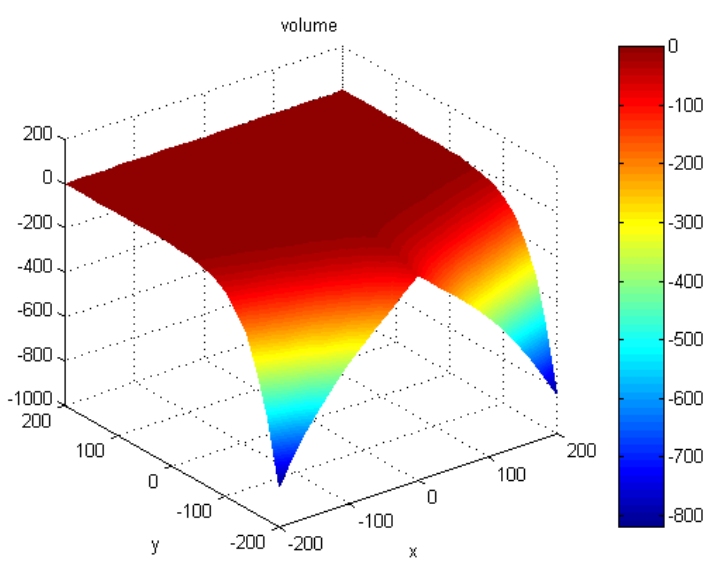

(b)

Figure 9 - profils de calibrage pour $\mathrm{h}=0$ (a) distribution de l'incertitude de mesure à la surface du podoscope.

- enfin, dans le cas du relevé d'escarre, on s'appuie sur des techniques de vision non calibrée et la reconstruction $3 \mathrm{D}$ est faite par stéréovision. La détection robuste de coins couleur appariés dans deux images est suffisante pour accéder à la géométrie 3D après estimation de la matrice fondamentale et un auto-calibrage valable pour une focale fixe. La précision finale reste inférieure à celle de systèmes calibrés mais une précision moyenne de $3 \%$ est suffisante pour la mesure de volume d'une escarre (Fig 10). Notons que l'auto-calibrage se dégrade fortement si les deux prises de vue sont en configuration orbitale. Pour obtenir des données métrologiques, il faut placer dans la scène un amer 
de longueur connue. Nous utilisons pour cela une petite tige terminée par une boule à chaque extrémité, que l'on place près de la plaie. En cas de projection de speckle, aucun calibrage supplémentaire n'est nécessaire puisque la lumière projetée ne sert pas directement à trianguler les coordonnées 3D, mais juste à faciliter la mise en correspondance des points homologues dans les images.

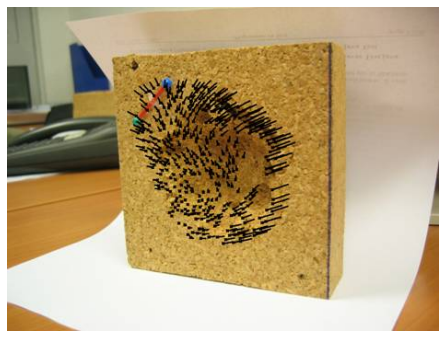

(a)

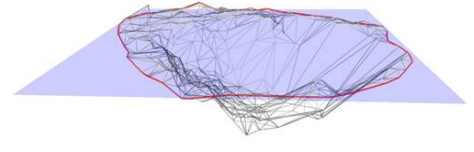

(b)

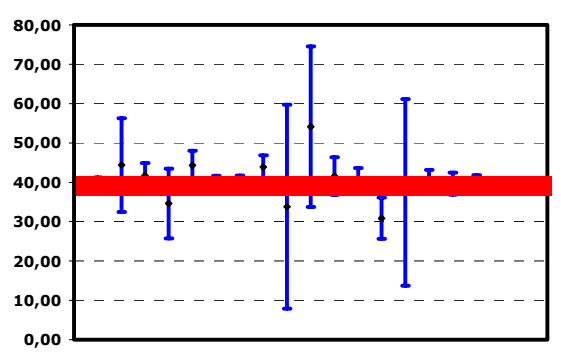

(c)

Figure 10 - objet test (a) bouchage du volume numérisé avec un plan (b) erreur moyenne et écart-type sur la mesure du volume.

\section{Détection de l'éclairage structuré}

La précision de la reconstruction 3D obtenue dépend de la qualité de la détection du trait dans l'image et du calibrage métrologique préalable du système. La détection des profils laser dans la séquence d'images doit optimiser trois critères : la robustesse, la précision et la rapidité. La détection des profils laser dans la séquence d'images peut-être effectuée hors-ligne pour ne pas pénaliser la durée d'un scan (risque de bougé) effectué avec une caméra CCD progressive voire avec une caméra CMOS rapide. Notons que le traitement d'image est grandement simplifié par l'adjonction sur l'objectif de la caméra d'un filtre interférentiel sélectif centré sur la longueur d'onde du laser.

La distribution radiale en amplitude d'un faisceau laser ponctuel est caractérisée par

$$
\mathrm{A}=\mathrm{A}_{0} \exp \left[-\mathrm{r}^{2} / \mathrm{w}^{2}\right]
$$

Où $\mathrm{A}_{0}$ désigne l'amplitude sur l'axe central, $\mathrm{r}$ le rayon et $\mathrm{w}$ un paramètre appelé taille du spot. Pour un faisceau plan, cette propriété reste valable mais s'ajoute une atténuation de l'intensité le long d'un profil (plus lumineux au centre que sur les bords) et l'étalement du trait suivant l'inclinaison du faisceau et l'orientation de la peau. La source laser produit une raie relativement indépendante de l'aspect de surface et permet le choix de seuils adaptatifs pour la détection de profil. La modélisation gaussienne précédente de l'intensité du trait laser permet une détection sub-pixel par ajustement d'une courbe paramétrée sur les données brutes (environ $10^{-2}$ pixels pour un trait d'épaisseur 5 pixels) [20]. Dans l'autre direction, le long du trait laser, on observe aussi une atténuation liée à l'étalement du faisceau source ponctuel, par une lentille cylindrique. Un seuillage adaptatif et des mires de calibrage avec dégradé de teintes sont recommandés. A titre d'exemple sur le podoscope, une caméra monochrome $640 \times 480$ pixels couplée à une diode laser et un moteur de $0.9^{\circ}$ d'angle par demi pas fournit un relevé plantaire de $10^{5}$ points. Des tests de répétabilité dénotent une incertitude de 0.15 pixel soit 0.1 à $0.3 \mathrm{~mm}$ mesurés 


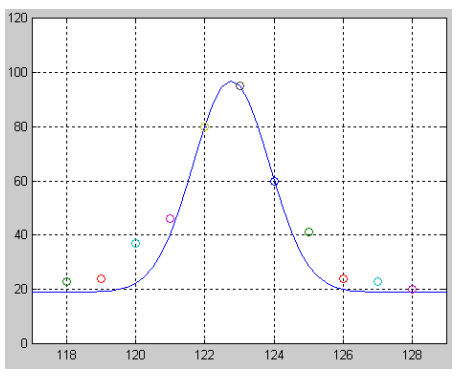

(c)
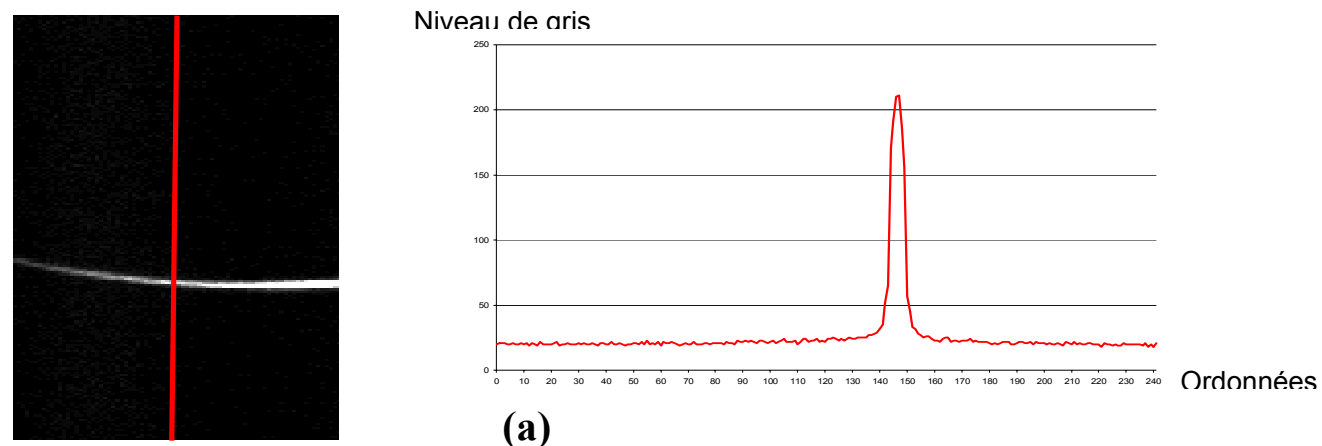

(a)

Figure 11 - détection du profil laser sur une colonne (a) profil d'intensité sur la colonne (b) ajustement gaussien de la courbe d'intensité (c)

Pour des patients à mobilité réduite (cas des porteurs d'escarres), on n'utilise pas de système à balayage laser mais un vidéo projecteur couplé à un appareil photo numérique. La luminosité et la résolution de l'appareil sont les deux critères importants pour générer une image de speckle de bonne qualité dont le motif aléatoire est généré par ordinateur (Fig.12).

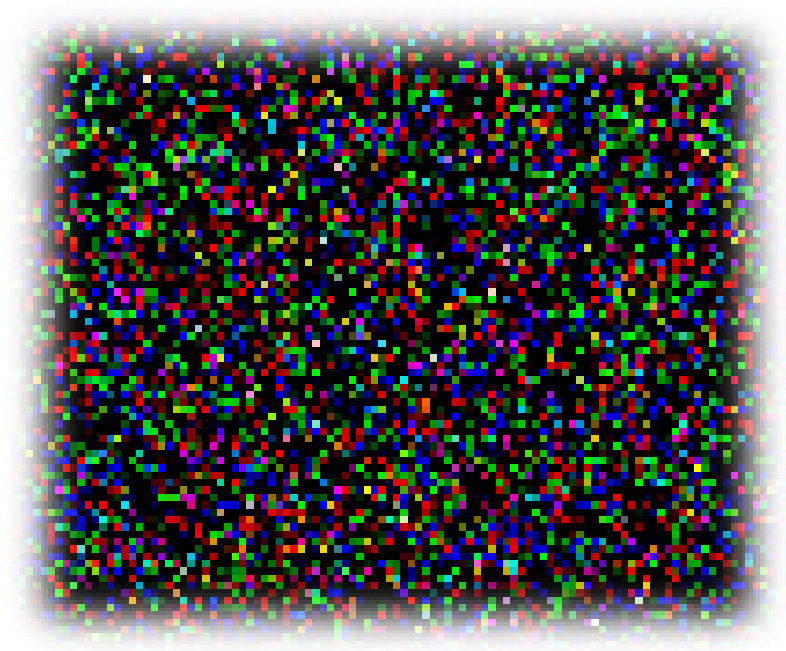

(a)

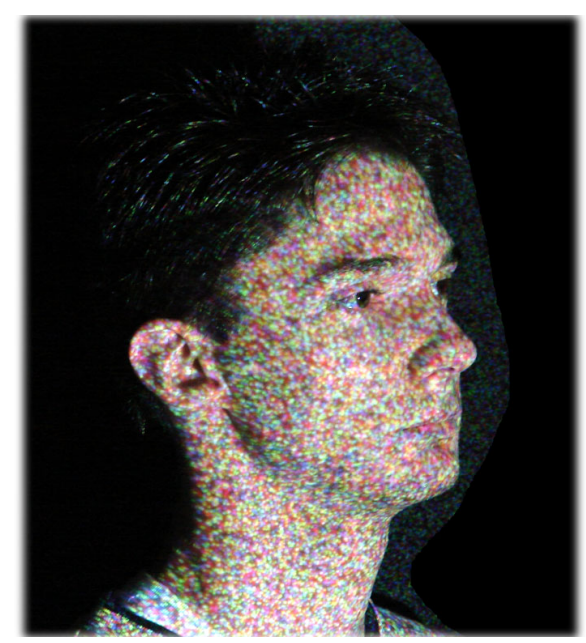

(b)

Figure 12 - motif texturé aléatoire (a) projection du motif sur la peau (b)

\section{Application biomédicale}

L'obtention de données brutes 3D décrivant la topographie de surface d'une région anatomique du corps humain ne sauraient constituer l'objectif final des relevés anatomiques. Le médecin peut disposer d'une réelle aide au diagnostic et d'un outil de mesure pour le suivi thérapeutique ou la conception d'orthèses personnalisées. Pour y parvenir, des connaissances expertes fournies par le médecin devront être introduites dans le système de mesure 3D. Des campagnes de mesures sont effectuées sur un grand nombre de patients pour lesquels le médecin va fournir un diagnostic permettant un apprentissage progressif. 
Par exemple, dans le cas du dépistage des scolioses, l'analyse de dissymétries dans le relevé 3D du rachis permet d'établir un pré-diagnostic en se basant sur le diagnostic médical obtenu par examen clinique et associé à un relevé 3D (Fig. 13a). Des campagnes en double aveugle permettent ensuite de juger de la fiabilité du pré-diagnostic (Fig. 13b) (taux de non-détection et de fausse détection des cas suspects).

Pour le suivi thérapeutique, c'est la comparaison entre deux relevés 3D effectués à des dates différentes, qui s'avère prépondérante. Il faut alors disposer d'outils de recalage automatique de surfaces pour en extraire des données quantitatives permettant d'évaluer un traitement : réduction d'une scoliose (dissymétrie atténuée, angulation vertébrale plus faible), rétablissement de la voûte plantaire (hauteur moyenne de l'arche), cicatrisation d'une escarre (volume interne réduit). Notons que dans le cas du diagnostic des escarres, le relevé 3D est couplé à une analyse couleur des tissus qui permet d'évaluer l'état de la plaie (Fig. 15).

L'obtention d'un relevé 3D permet également la conception d'orthèses (Fig. 14) mais celle-ci sera réservée au spécialiste. Pour réaliser une semelle correctrice monobloc, il faut déformer le relevé 3D brut en ajoutant des compensations : c'est ce que fait traditionnellement le podologue lorsqu'il ajoute des cales de forme et de tailles spécifiques sur une semelle brute. De même, il ne suffit pas d'avoir un relevé 3D pour confectionner un corset. Des compensations devront être ajoutées sur la forme brute pour induire localement des surpressions destinées à redresser la colonne vertébrale. Ce modelage du relevé $3 \mathrm{D}$ brut se fera en $\mathrm{CAO}$ de façon interactive par le prothésiste.

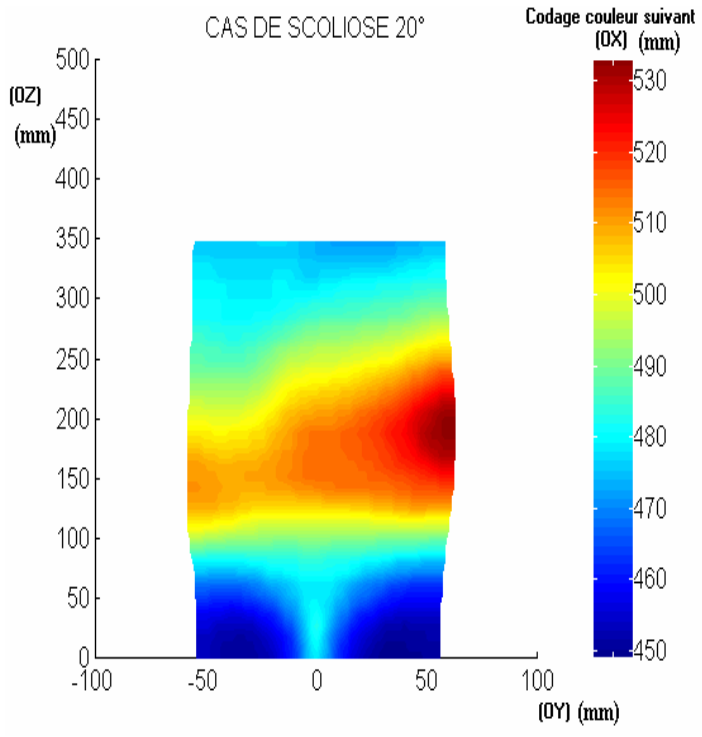

(a)

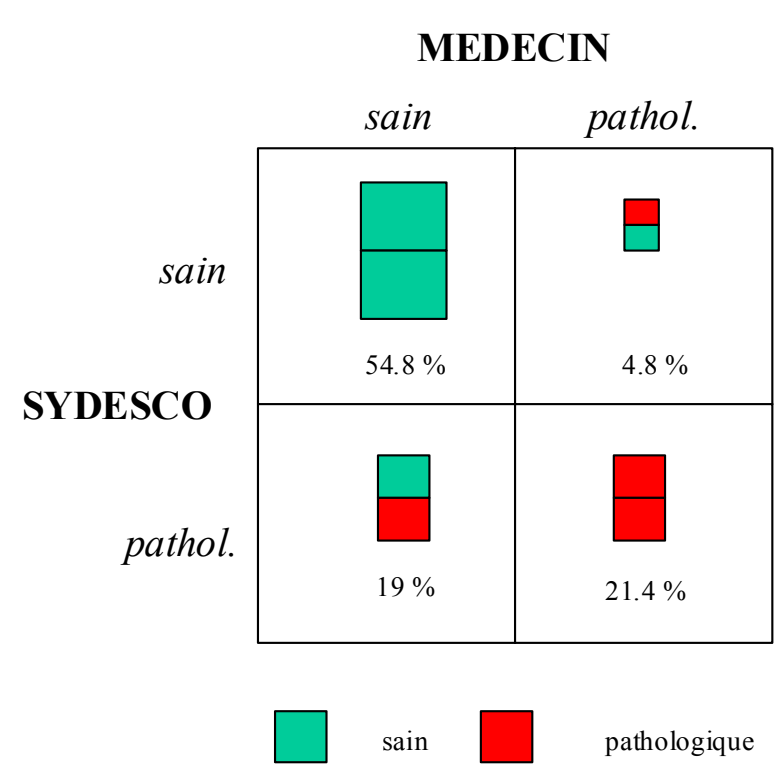

(b)

Figure 13 - relevé 3D d'un rachis scoliotique (a) évaluation du dépistage automatique (b) 


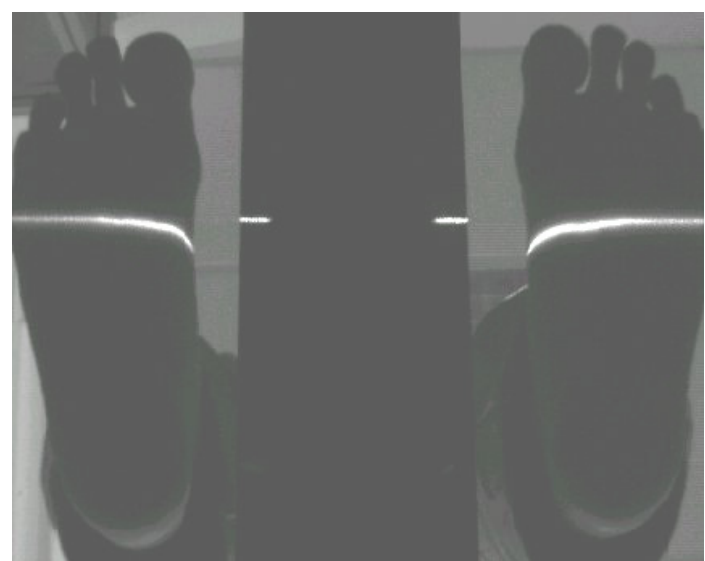

(a)

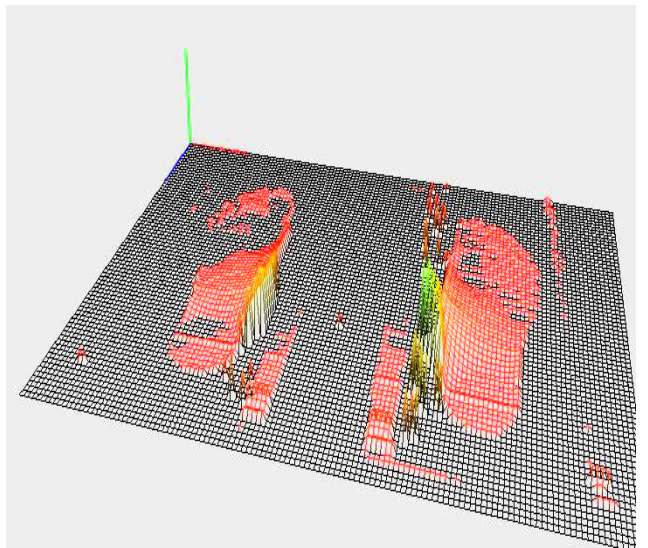

(b)

Figure 14 - relevé d'empreinte plantaire sur podoscope (a) visualisation sous Open GL (b)

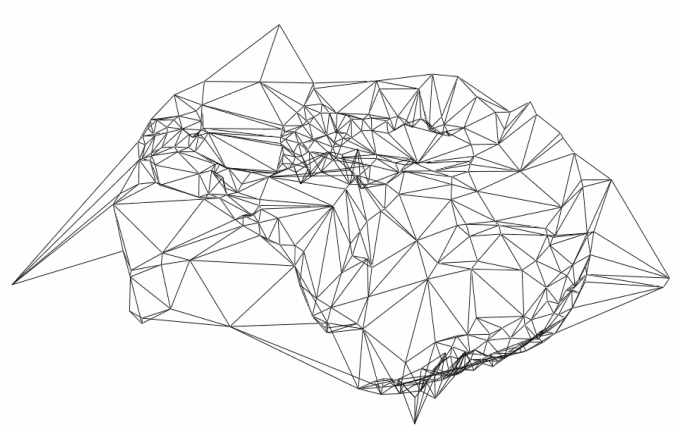

(a)

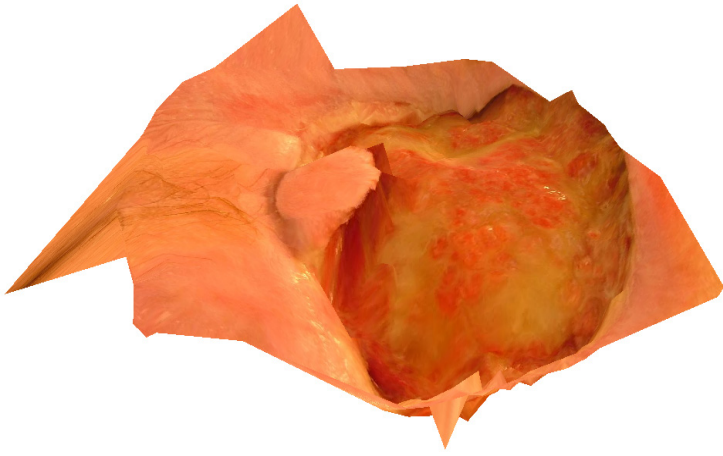

(b)

Figure 15 - fichier STL du relevé 3D (a) relevé 3D avec placage de la texture naturelle (b)

\section{Conclusion}

La vision active, parvenue à maturité grâce aux avancées technologiques sur les imageurs, l'optoélectronique et la vidéo projection, se prête particulièrement bien au relevé anatomique de parties du corps humain. Elle permet d'obtenir rapidement et sans contact une topographie de surface, obtenue traditionnellement par des procédés manuels, lents, coûteux et peu précis tels que le moulage. La numérisation permet en outre la télétransmission des mesures et leur comparaison. Nous avons présenté ici des applications dans le domaine de la santé, développées dans le cadre du programme RAVIR : dépistage des scolioses, conception de semelles personnalisées et suivi thérapeutique d'escarres. Les étapes successives de la conception d'un système de vision active appliqué au médical ont été présentées au travers de ces exemples, en exposant la spécificité de chaque système et les aspects communs liés à la techniques de vision active. Le relevé anatomique ne se limite cependant pas au secteur médical, comme en témoigne la récente campagne nationale de relevé 3D complet du corps humain, destinée à évaluer l'évolution de la morphologie pour l'industrie de l'habillement.

Remerciements : le programme RAVIR bénéficie de soutiens de la Région Centre, de la Délégation Régionale à la Recherche et Technologie et des Fonds Sociaux Européens. 


\section{Bibliographie}

[1] S. Pankanti and A. Jain "Biometrics : the future of identification" Computer, Vol. $33 \mathrm{n}^{\circ} 2,2000$, p. 46-49.

[2] U. Dieckmann, P. Plankensteiner and T. Wagner "SESAM : a biometric person identification system using sensor fusion" Pattern recognition letters, Vol. 18 n9, 1997, p.827-833.

[3] G. Hutton, M. Bayley, M. Broadhead and A. Knox "human shape analysis : applications and methods in clothing applications", Proc. of Scanning 2002 Conf. on Human Modelling, Paris 24-25/04 2002.

[4] Hamamatsu Corp. "invasion of the body scanners" IEEE Circuits and Devices magazine, Vol. 13 n³, 1997, p.12-17.

[5] K.M.Robinette, H. Daanen and E. Paquet "the Caesar project : a 3-D surface anthropometry survey " Proc. of the $2^{\text {nd }}$ Int. Conf. on 3-D Digital Imaging and Modeling, 4-8 Oct 1999, Ottawa Canada p.380386.

[6] S. Treuillet, Y. Lucas, G. Crépin, B. Peuchot and J.C. Pichaud "Sydesco : A laser-video scanner for 3-D scoliosis evaluations ", Research into Spinal Deformities 3- Studies in Health Technology and Informatics, IOS Press Amsterdam, 2002, p.70-73.

[7]Y.Lucas, S.Treuillet,J.C. Pichaud "A plantar arch 3D scanner for the conception of orthopaedic soles" , $8^{\text {th }}$ Int. Conf. on 3D modelling-3D Medical, Paris 23-24 /04/2004, p.10-16.

[8] B.Albouy, S.Treuillet, Y.Lucas, J.C.Pichaud, "Volume Estimation from uncalibrated views applied to wound measurement", $13^{\text {th }}$ Int. Conf. on Image Analysis and Processing, Cagliari 6-8 Sept. 2005 Italy [9] M. Kouchi and M. Mochimaru, "Development of a low cost foot-scanner for a custom shoe making system" Proc. of the $5^{\text {th }}$ Symposium on Footwear Biomechanic, Zurich/switzerland, Eds. E.Henning, A.Stacoff, 2002, p.58-59.

[10] P.Plassman, T.D.Jones "MAVIS : a non-invasive instrument to measure area and volume of wounds“, Med. Eng. Phys. Vol.20 n5, 1998, p.332-338.

[11] S.M. Boersma \& al "Phtogrammetric wound measurement with a three-camera vision system", Int. Archives of Photogrammetry and Remote Sensing, Vol. 33, Amsterdam , 2000.

[12] A. Malian \& al "A robust photogrammetric system for wound measurement", Int. Archives of Photogrammetry and remote sensing, Vol. 34, Part 5, 2002,p.264-269.

[13]T.A. Krouskop \& al "A non contact wound measurement system", Journal of Rehabilitation R\&D, Vol.39, n³, May/June 2002, p.337-346.

[14] J. Pagès, J.Salvi, R. Garcia and C.Matabosch "Overview of coded light projection techniques for automatic 3D profiling", Proc. of the 2003 IEEE Int. Conf. on Robotics and Automation, 2003, September 14-19, 2003, Taipei, Taiwan. p.133-138.

[15] J.P. Siebert, S.J.Marshall "Human body 3D imaging by speckle texture projection photogrammetry “, Sensor Review, MCB University Press, Vol.20 n³ , 2000, p.218-226.

[16] R.Y. Tsai "An efficient and accurate camera calibration technique" Proc. of IEEE. Conf. on Computer Vision and Pattern Recognition, Miami Beach Florida USA, 1986, p.364-374.

[17] B. Tiddeman, N. Duffy, G. Rabey, and J. Lockier "Laser-video scanner calibration without the use of a frame store "IEE Proc. Image Signal Processing, Vol. 145 n4, August 1998.

[18] E. Trucco and R.B. Fisher "Acquisition of consistent range data using direct calibration" Proc. of IEEE Int. Conf. On robotics and automation. San Diego, California, 1994, p.3410-3415.

[19] B. Peuchot "Camera virtual equivalent model 0.01 pixel detectors" Computerized medical imaging and graphics, 1993, Vol.17 n4-5, p.289-294.

[20] D.K. Naidu and R. Fisher " A comparative analysis of algorithms for determining the peak position of a stripe to subpixel accuracy" Proc. of British Machine Vision Conf. ,1991, p.217-225. 